\title{
Systems Biology: Models and Logics ${ }^{\star}$
}

\author{
C. Piazza ${ }^{1}$ and A. Policriti ${ }^{1,2}$ \\ ${ }^{1}$ Dept. of Math. and Computer Science, University of Udine, Udine, Italy \\ ${ }^{2}$ Institute of Applied Genomics, Udine, Italy \\ \{piazza, policriti\}@dimi. uniud.it
}

\begin{abstract}
The field of systems biology focuses on creating a finely detailed picture of biological mechanisms. Recently, the need has arisen for more and more sophisticated and mathematically well founded computational tools, capable of analyzing those models that are and will be at the core of Systems Biology. The challenge consists in faithfully implementing such computational models in software packages exploiting the potential trade-offs among usability, accuracy, and scalability when dealing with large amounts of data. The aim of this presentation is that of introducing some emerging problems and proposed solutions in this context.

In particular, after an introductory first part, in the second part we will focus on the use of Hybrid Systems in Systems Biology. Hybrid systems are dynamical systems presenting both discrete and continuous evolution, originally proposed to study embedded systems, where a discrete control acts on a continuously changing environment. The presence of both discrete and continuous dynamics makes this formalism appealing also for modeling biological systems. However, the situation in this case is subtler, basically because there is no "natural" separation of discrete and continuous components. It comes as no surprise, therefore, that Hybrid Systems have been used in Systems Biology in rather various ways. Some approaches, like the description of biological switches, concentrate on the use of model-checking routines. Other applications, like the switching between continuous and discrete/stochastic simulation, focus on the exploitation of the interplay between discreteness and continuity in order to reduce the computational burden of numerical simulation, yet maintaining an acceptable precision. We will survey some of the main uses of Hybrid Automata in Systems Biology, through a series of cases studies that we deem interesting and paradigmatic, discussing both actual and foreseeable, logical and implementation issues.
\end{abstract}

\footnotetext{
* This work is partially supported by PRIN "BISCA" 2006011235 and FIRB "LIBI" RBLA039M7M.
} 